\title{
Stewardship program on carbapenem prescriptions in a tertiary hospital for adults and children in France: a cohort study
}

\author{
Julie Poline ${ }^{1,2}$ (I) Martine Postaire ${ }^{3}$ - Perrine Parize ${ }^{4} \cdot$ Benoit Pilmis $^{4} \cdot$ Emmanuelle Bille $^{5} \cdot$ Jean Ralph Zahar ${ }^{5,6}$. \\ Pierre Frange ${ }^{5}$ • Jérémie F. Cohen ${ }^{1} \cdot$ Olivier Lortholary $^{4} \cdot$ Julie Toubiana ${ }^{1,7}$
}

Received: 16 September 2020 / Accepted: 5 November 2020 / Published online: 3 January 2021

(C) Springer-Verlag GmbH Germany, part of Springer Nature 2021

\begin{abstract}
Antimicrobial stewardship programs aim at reducing the overuse of broad-spectrum antibiotics such as carbapenems, but their impact remains unclear. We compared the use of carbapenems between paediatric and adult subjects admitted to a French tertiary hospital and described the intervention of an antibiotic stewardship team (AST). As part of AST routine activity, all adult and paediatric patients receiving carbapenems are identified in real time using a computergenerated alert system and reviewed by the AST. Data associated with carbapenem prescriptions were extracted for 2 years (2014-2015) and were compared between paediatric and adult wards. Prescription appropriateness (i.e. no clinically suitable narrower spectrum alternative to carbapenem for de-escalation) and AST intervention were analysed. In total, 775 carbapenem prescriptions for 291 children and 262 adults were included. Most patients $(95 \%)$ had a comordity and 52\% had known recent carriage of extended-spectrum beta-lactamase producing Enterobacteriaceae (ESBLE). Most carbapenem prescriptions came from intensive care units $(n=269,35 \%)$ and were initiated for urinary tract $(n=200,27 \%)$, sepsis $(n=181,25 \%)$, and lung $(n=153,21 \%)$ infections. Carbapenems were initiated empirically in $537(70 \%)$ cases, and an organism was isolated in $523(67 \%)$ cases. Among the isolated organisms, $47 \%(n=246)$ were ESBLE and $90 \%(n=468)$ were susceptible to carbapenems, but an alternative existed in $61 \%(n=320)$ of cases according to antibiotic susceptibility testing. Among prescriptions reviewed by the AST, 39\% $(n=255)$ were considered non-appropriate and led to either antibiotic discontinuation $(n=47,7 \%)$ or de-escalation $(n=208,32 \%)$. Non-appropriate prescriptions were more frequent in paediatric wards $(p=0.01)$ and in microbiologically documented infections $(p=0.013)$, and less observed in immunocompromised patients $(p=0.009)$ or with a known ESBLE carriage $(p<0.001)$. Tailored stewardship programs are essential to better control carbapenem use and subsequent antimicrobial resistance.
\end{abstract}

Keywords Carbapenems · Anti-infective agents · Antimicrobial stewardship · Extended-spectrum beta-lactamase Enterobacteriaceae

Julie Toubiana

julie.toubiana@aphp.fr

1 Department of General Paediatrics and Paediatric Infectious Diseases, Hôpital Necker-Enfants Malades, APHP, Université de Paris, 149 rue de Sèvres, 75015 Paris, France

2 Department of Gut Inflammation, Center for Research on Inflammation CRI, INSERM 1149, Université de Paris, 75006 Paris, France

3 Department of Pharmacy, Hôpital Necker-Enfants Malades, APHP, Université de Paris, Paris, France
4 Department of Infectious Diseases and Tropical Medicine, Necker Enfants-Malades Hospital, Necker-Pasteur Infectious Diseases Center, Université de Paris, IHU Imagine, Paris, France

5 Department of Microbiology, Necker Enfants-Malades Hospital, APHP, Université de Paris, Paris, France

6 Infection Control Unit, IAME, UMR 1137, Université Paris 13, Sorbonne Paris Cité, Paris, France

7 Biodiversity and Epidemiology of Bacterial Pathogens, Institut Pasteur, Paris, France 


$\begin{array}{ll}\text { Abbreviations } \\ \text { AST } & \begin{array}{l}\text { Antimicrobial stewardship team } \\ \text { Extended-spectrum beta-lactamase } \\ \text { ESBLE }\end{array} \\ \text { EUCAST } & \begin{array}{l}\text { European Committee on } \\ \text { Antimicrobial Susceptibility Testing }\end{array} \\ \text { ICU } & \begin{array}{l}\text { Intensive care unit } \\ \text { NICU }\end{array} \\ \text { NK/PD } & \begin{array}{l}\text { Pharmacolal intensive care unit } \\ \text { Interquartile }\end{array} \\ \text { IQR } & \text { pharmacodynamics }\end{array}$

\section{Introduction}

Infections with multidrug-resistant bacteria are an emerging threat worldwide. Inappropriate use of antibiotics and subsequent selective pressure might participate in spreading antimicrobial resistance. As observed in other European countries, the incidence of extended-spectrum beta-lactamase producing Enterobacteriaceae (ESBLE) infections has seen a fourfold increase between 2006 and 2016 in France [1, 2]. A significant increase has also been observed in the paediatric community [3]. ESBLE infections can cause failure of classic antibiotic therapy strategies and might require the use of carbapenems, which are considered the most effective antibiotic agents against ESBLE-related infections [4-6].

Facing the increasing prevalence of ESBLE carriage and subsequent risk of ESBLE infection [7-10], carbapenems are sometimes prescribed without any microbiological documentation, particularly in settings at high risk of antimicrobial resistance such as intensive care units (ICU), which might lead to carbapenem overuse [11]. Empirical broad-spectrum antibiotic therapy has critical ecological consequences because of the emergence of bacterial resistance. Indeed, even a short exposure to carbapenem is considered a major risk factor of carbapenem-resistant Gram-negative bacilli intestinal carriage [12].

In France, a twofold increase in carbapenem use has been observed between 2000 and 2015 [13, 19]. Monitoring of over- and inappropriate carbapenem use is, besides regular surveillance of multidrug resistance prevalence, crucial to define corrective actions. Antimicrobial stewardship programs and antimicrobial stewardship teams (AST) were created in many countries, and several studies have shown their effectiveness in reducing inappropriate antimicrobial use and hospital costs on antimicrobials, rates of antimicrobial resistance, and in improving patient care and outcomes [14-20]. However, few data are available regarding the modalities of carbapenem use and the acceptance of AST interventions in paediatric wards [21-24], and no study has compared paediatric and adult carbapenem use.

A review of carbapenem prescriptions with real-time monitoring and intervention of AST was formally implemented in our university hospital in 2013. To better understand carabapenem indications and appropriateness, and the impact of an AST intervention in a large tertiary hospital, we analysed all carbapenem prescriptions over a 2-year period, in both paediatric and adult cases.

\section{Patients and methods}

\section{Study design and setting}

The Necker-Enfants Malades Hospital is a large university hospital in the centre of Paris, France, and includes paediatric and adult wards. This hospital has set an antimicrobial surveillance program for carbapenems together with the Pharmacy Department in 2013. This program is held by the AST, a multisciplinary team consisting of three part-time adult and paediatric infectious disease specialists, a clinical microbiologist, and a pharmacist. In our hospital, AST daily advises physicians (approx. 15 consultations a day), in terms of diagnosis, therapeutics, and prevention, and systematically visits critical settings such as ICUs and Neonatal ICUs (NICUs). AST is informed by the hospital pharmacy of any new carbapenem prescription within the next $24-48 \mathrm{~h}$ using a computergenerated alert system. All alerts lead to contact with the prescriber to discuss the case and the relevance of carbapenem use. All clinical, biological, and pharmacological data associated with AST activity are routinely collected in a database.

We retrospectively analysed all carbapenem prescriptions between January 1, 2014, and December 31, 2015. The intervention of the AST was collected and analysed for each prescription. It included carbapenem discontinuation, discontinuation of any other antibiotic therapy, switch to a narrower spectrum antimicrobial, optimal or non-optimal dosing, and consideration of further diagnostic workup.

\section{Data collection and microbiology}

For each carbapenem prescription, the AST collected the following data: patient demographics, pre-existing illness such as cancer or transplant, renal impairment, previous colonization or infection with an ESBLE within the last 3 months (ESBLE carriage), characteristics of the infection which motivated the prescription of carbapenem, and the type of pathogen (if the episode was documented) and its antibiotic susceptibility. ESBLE detection and in vitro susceptibility testing involved a double-disk synergy test between clavulanic acid and extended-spectrum cephalosporin (ceftazidime and ceftriaxone) and the disk diffusion method, respectively, as recommended by the European Committee on Antimicrobial Susceptibility Testing (EUCAST) [25]. 


\section{Definitions}

A carbapenem prescription was defined as a course of carbapenem therapy for a certain indication in a certain continuous time range without disruption (as from the first administration). Patients with carbapenem prescription were either from paediatric wards ( 400 beds) that consisted in departments of Medicine (general paediatrics, neurology, endocrinology, gastroenterology, pneumology, dermatology, nephrology, haematology), departments of Surgery, and paediatric and neonatal ICU, or adult wards ( 200 beds) that consisted in departments of infectious diseases, nephrology and transplantation, haematology, and adult ICU). Each patient registered at the wards during the daily registration was regarded as one patient day. Treatment duration was defined as number of days with carbapenem exposure. Carbapenem days of therapy (DOT) was calculated and standardized to 1000 patient days [26]. Invasive infection was defined according to the US Centers for Disease Control and Prevention [27]. Sepsis and septic shock were defined according to the international guidelines available at the time of the study [28]. Carbapenem dosage and administration were considered optimal if they followed the local guidelines according to renal function and met requirements on the basis of available pharmacokinetic/pharmacodynamics (PK-PD) evidence for carbapenems, i.e. dosing interval and duration of infusion [29]. Carbapenem prescription was defined as appropriate if the members of the AST in charge of the case (at least one resident and one senior infectious disease specialist) considered that, at the time of the prescription review, there was no clinically suitable narrower spectrum alternative to carbapenem antibiotics for de-escalation [30] based on their individual expertise and international and national standards (e.g. documented infections without any alternative to carbapenem, empirical therapy to patients with septic shock and considered to be at high risk for ESBLE) [31]. Microbiologically effective antimicrobial treatment against the causative pathogen was defined as adequate [29]. ESBLE carriage was defined as a history of ESBLE rectal colonization or infection in the last 6 months.

\section{Statistical analysis}

Chi-square and Mann-Whitney $U$ tests were used to compare the distributions of patients', infections', and prescriptions' characteristics between adult and paediatric carbapenem prescriptions and between appropriate and non-appropriate carbapenem prescriptions, using SPSS v21 (SPSS Inc., Chicago, IL). A $p$ value was considered statistically significant if $<0.05$ (two-sided). Variables associated with non-appropriate carbapenem prescriptions were analysed using logistic regression. Odds ratios and $95 \%$ confidence intervals $(95 \% \mathrm{CI})$ were estimated in univariate analysis.

\section{Ethical approval}

This study was reviewed and approved by the Necker Hospital Institutional Review Board (Registration number in the registry of the Assistance Publique-Hôpitaux de Paris: 2020 0117181406). All data processing and storage comply with the General Data Protection Regulation (GDPR) and ethical standards of the National Research Committee.

\section{Results}

\section{Characteristics of patients and prescriptions}

In total, 803 carbapenem prescriptions were identified during the study period, 775 of them had available and comprehensive data for further analyses, which concerned 553 patients (291 children and 262 adults). Regarding the 775 included prescriptions, 405 came from paediatric wards and 370 from adult wards (Table 1), with a median patient age of 1.6 [IQR, 0.3-8.8] years and 61.6 [IQR, 46.6-70.2] years, respectively. The male/female sex ratio was 1.1 and did not differ between adult and paediatric wards. The majority of patients under carbapenems had comorbidities (95\%), including 57\% $(n=$ 303) patients with an immune deficiency (i.e. solid organ or haematopoietic stem cell transplantation, malignancy, inherited immune deficiency including sickle cell disease, and immunosuppressive treatment), $22 \%(n=124)$ with digestive, neurological, urological, or respiratory chronic disorders, and $11 \%(n=61)$ with cardiac failure. Nineteen children (2.4\%) were preterm infants. Renal impairment was present in 151 and 41 patients from adult and paediatric wards, respectively.

Documented ESBLE colonization prior to carbapenem prescription was found in $52 \%$ of the 747 cases with available data, $55 \%$ in paediatric, and $50 \%$ in adult wards (Table 1). Patients with immune deficiency had a higher risk of carrying ESBLE prior to carbapenem prescription (56\% vs. $44 \%, p=$ 0.009).

Adult departments of medicine combining nephrology and infectious disease wards were the main prescriptors of carbapenems, followed by paediatric ICUs (Table 1). Imipenem was the most prescribed carbapenem in both paediatric $(n=$ $205,51 \%)$ and adult cases $(n=219,59 \%)$; but the proportion of molecules was different between adults and children $(p<0.001)$. We did not find any statistically significant difference in treatment duration between adult and paediatric wards, with a median of 7 [IQR, 3-13] and 5 [IQR, 3-10] days, respectively. When the number of days of carbapenem prescription (DOT) was standardized to 1000 patient days, carbapenem use was higher in adult (36.1/1000 patient days) than in paediatric wards (12.4/1000 patient days). The highest standardized carbapenem use was observed in the ICUs, followed 
Table 1 Characteristics of included carbapenem prescriptions $(N=775)$

\begin{tabular}{|c|c|c|c|c|}
\hline$N(\%)$, otherwise stated & Total & Paediatric prescriptions & Adult prescriptions & $p^{*}$ \\
\hline Wards prescribing carbapenems & $n=775$ & $n=405$ & $n=370$ & $<0.001$ \\
\hline Departments of medicine & $311(40)$ & $88(22)$ & $223(60)$ & \\
\hline Departments of surgery & $32(4)$ & $32(8)$ & N/A & \\
\hline Intensive care units & $269(35)$ & $199(49)$ & $70(19)$ & \\
\hline Departments of haematology/immunology & $110(14)$ & $33(8)$ & $77(10)$ & \\
\hline Neonatal intensive care unit & $53(7)$ & $53(13)$ & N/A & \\
\hline Molecule & & & & $<0.001$ \\
\hline Ertapenem & $23(3)$ & $6(1.5)$ & $17(5)$ & \\
\hline Imipenem & $424(55)$ & $205(51)$ & $219(59)$ & \\
\hline Meropenem & $328(42)$ & $194(48)$ & $134(36)$ & \\
\hline Duration, days: median (IQR) & $6(3-11)$ & $5(3-10)$ & $7(3-13)$ & 0.05 \\
\hline Empirical prescription & $537(70)$ & $285(71)$ & $252(72)$ & 0.49 \\
\hline Optimal prescription according to recommendations & $n=533$ & $n=283$ & $n=250$ & \\
\hline Dosage & $469(88)$ & $264(93)$ & $205(82)$ & 0.01 \\
\hline Administration & $481(90)$ & $264(93)$ & $217(87)$ & 0.03 \\
\hline ESBLE screening & $n=747$ & $n=391$ & $n=356$ & \\
\hline Known ESBLE carriage & $392(52)$ & $213(55)$ & $179(50)$ & 0.02 \\
\hline Site of infection & $n=738$ & $n=377$ & $n=361$ & $<0.001$ \\
\hline Lower respiratory tract infection & $153(21)$ & $96(26)$ & $57(16)$ & \\
\hline Urinary tract infection & $200(27)$ & $73(19)$ & $127(35)$ & \\
\hline Deep infection & $82(11)$ & $48(13)$ & $34(9)$ & \\
\hline Central nervous system & $10(1)$ & $5(1)$ & $5(1)$ & \\
\hline Superficial infection ${ }^{* *}$ & $21(3)$ & $8(2)$ & $13(4)$ & \\
\hline Surgical prophylaxis & $19(3)$ & $17(5)$ & $2(1)$ & \\
\hline Febrile aplasia & $72(10)$ & $29(8)$ & $43(13)$ & \\
\hline Sepsis without identifiable infection & $181(25)$ & $101(27)$ & $80(22)$ & \\
\hline Bacteraemia & $82(11)$ & $41(11)$ & $41(11)$ & 0.84 \\
\hline Septic shock & $66(9)$ & $34(9)$ & $32(9)$ & 0.94 \\
\hline Healthcare-associated infection & $620(84)$ & $327(91)$ & $293(78)$ & 0.16 \\
\hline
\end{tabular}

${ }^{*} p$ value of the $\mathrm{Chi}^{2}$ or Mann-Whitney test between paediatric and adult carbapenem prescriptions.* Surgical site or skin infections

by the units of Haematology/Immunology (Fig. 1). Carbapenems were mainly initiated empirically in both paediatric and adult wards (70\% vs. 71\%, respectively) (Table 1). Empirical treatment was more frequent in immunocompromised patients ( 77 vs. $70 \%, p=0.03$ ), but no difference was observed according to the status of ESBLE carriage or the type of ward. The dosage and administration of carbapenem were more often optimal in paediatric wards (93\% vs. $82 \%$, $p=0.01$, and $93 \%$ vs. $82 \%, p=0.03$ ).

\section{Characteristics of infections and microbiological data}

Among the 738 prescriptions with available data, treatment was mostly initiated for urinary tract infections $(n=200$, $27 \%)$, sepsis $(n=181,25 \%)$, and lung infections $(n=153$, $21 \%$ ) (Table 1). Septic shock was observed in $9 \%$ of the cases $(n=66)$. Healthcare-associated infections represented $84 \%$ of the cases and occurred almost exclusively in patients with comorbidities $(95 \%)$.

Causative pathogens were documented in 523 cases (Table 2). Among documented infections, carbapenems were initiated after documentation in $37 \%$ of cases $(n=196)$. Klebsiella spp. were the main pathogen involved. ESBLE were identified in $47 \%$ of cases $(n=246)$. Nocardia spp. and $E$. coli were more frequent in adult wards, while Klebsiella spp. and Pseudomonas spp. were more observed in paediatric wards. Most of the identified pathogens were susceptible to carbapenems (90\%), and there was an alternative to carbapenems in $61 \%$ of cases according to antibiotic susceptibility testing (mainly cephamycins and other beta-lactams). No difference in terms of antibiotic treatment adequacy according to antibiotic susceptibility was found between paediatric and adult wards. Among non-documented infections, febrile aplasia $(25 \%)$ and septic shock (19\%) were the main causes 


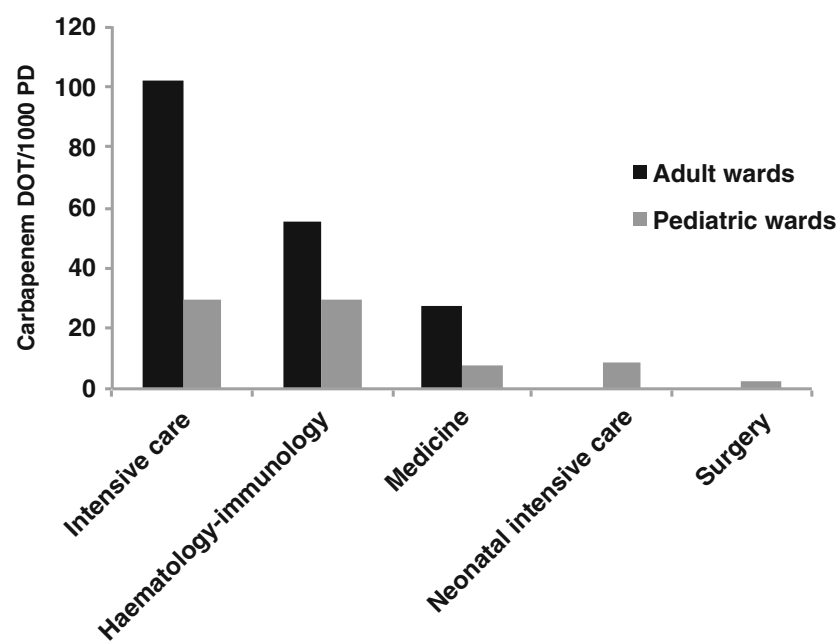

Fig. 1 Days of carbapenem therapy according to wards. DOT/1000 PD, days of therapy per 1000 patient days

of carbapenem initiation, and $38 \%$ and $43 \%$ of the corresponding patients had a history of ESBLE carriage, respectively.

\section{AST intervention}

Among the 775 prescriptions, 688 (90\%) were reviewed by AST and 658 of them had available and comprehensive data for further analyses (Fig. 2 and Table 3). The median response time of AST after carbapenem initiation was 3.5 (range, $0-30)$ days. Among the 255 (39\%) non-appropriate prescriptions, $82 \%$ of them had a suitable alternative for de-escalation, such as $3 \mathrm{rd}$ generation cephalosporin $(n=55)$, piperacillin-tazobactam $(n=40)$, cotrimoxazole $(n=17)$, cefepime $(n=15)$, fluoroquinolones $(n=12)$, or cefoxitin $(n=8)$. The proportion of non-appropriate prescriptions remained stable between 2014 (38.8\%) and 2015 (38.7\%).

In univariate analysis, non-appropriate prescriptions were more often observed within paediatric wards compared to adult wards $(\mathrm{OR}=1.5 ; 95 \% \mathrm{CI}=1.1-2.1)$ and in documented infections $(\mathrm{OR}=1.6 ; 95 \% \mathrm{CI}=1.1-2.2)$. Cases with nonappropriate prescriptions were less likely to have a previous ESBLE carriage $(\mathrm{OR}=0.5,95 \% \mathrm{CI}=0.4-0.7)$, an immune deficiency $(\mathrm{OR}=0.7,95 \% \mathrm{CI}=0.5-0.9)$, and an infection caused by ESBLE $(\mathrm{OR}=0.1,95 \% \mathrm{CI}=0.08-0.2)$ or complicated by septic shock $(\mathrm{OR}=0.6 ; 95 \% \mathrm{CI}=0.3-0.9)$ (Table 3$)$. The site of infection was not associated with carbapenem appropriateness $(p=0.07)$.

\section{Discussion}

\section{Main findings}

The increasing use of carbapenems observed worldwide prompt us to better monitor their prescriptions in our hospital. In this study, carbapenems were used more often in adult than paediatric wards and were generally initiated in severely ill patients having serious comorbidities and/or risk factors of drug resistance. Around $50 \%$ of patients under carbapenem regimen had a known carriage of ESBLE, which might have been underestimated, as some patients have not been systematically screened for carriage within the first days of hospitalization and rectal swab has false-negative results [32]. Carbapenems were often prescribed empirically $(73 \%)$

Table 2 Documented infections associated with carbapenem prescriptions $(N=523)$

\begin{tabular}{|c|c|c|c|c|}
\hline Characteristics of documented infections, $N(\%)$ & $\begin{array}{l}\text { Total } \\
n=523\end{array}$ & $\begin{array}{l}\text { Paediatrics prescriptions } \\
n=265\end{array}$ & $\begin{array}{l}\text { Adult prescriptions } \\
n=258\end{array}$ & $p$ \\
\hline Carbapenem treatment initiation following documentation & $196(37)$ & $98(37)$ & $98(38)$ & 0.81 \\
\hline ESBLE & $246(47)$ & $124(47)$ & $122(47)$ & 0.91 \\
\hline Agents & & & & 0.01 \\
\hline Klebsiella spp. & $151(29)$ & $89(34)$ & $62(24)$ & \\
\hline Enterobacter spp./Citrobacter koseri & $104(20)$ & $53(20)$ & $51(20)$ & \\
\hline E. coli & $116(22)$ & $46(17)$ & $70(27)$ & \\
\hline Nocardia spp. & $12(2)$ & $2(1)$ & $10(4)$ & \\
\hline Pseudomonas spp. & $75(14)$ & $47(18)$ & $28(11)$ & \\
\hline Gram-positive cocci & $64(12)$ & $38(14)$ & $26(10)$ & \\
\hline Other & $63(12)$ & $42(16)$ & $21(8)$ & \\
\hline \multicolumn{5}{|l|}{ Antibiotic therapy } \\
\hline Adequate $^{\#}$ & $468(90)$ & $236(89)$ & $232(90)$ & 0.65 \\
\hline Existing alternative to carbapenems ${ }^{\#}$ & $320(61)$ & $173(65)$ & $147(57)$ & 0.15 \\
\hline
\end{tabular}

${ }^{\#}$ Microbiologically effective according to EUCAST antibiotic susceptibility testing; ${ }^{*} p$ value of the Chi ${ }^{2}$ or Mann-Whitney test between paediatric and adult wards 


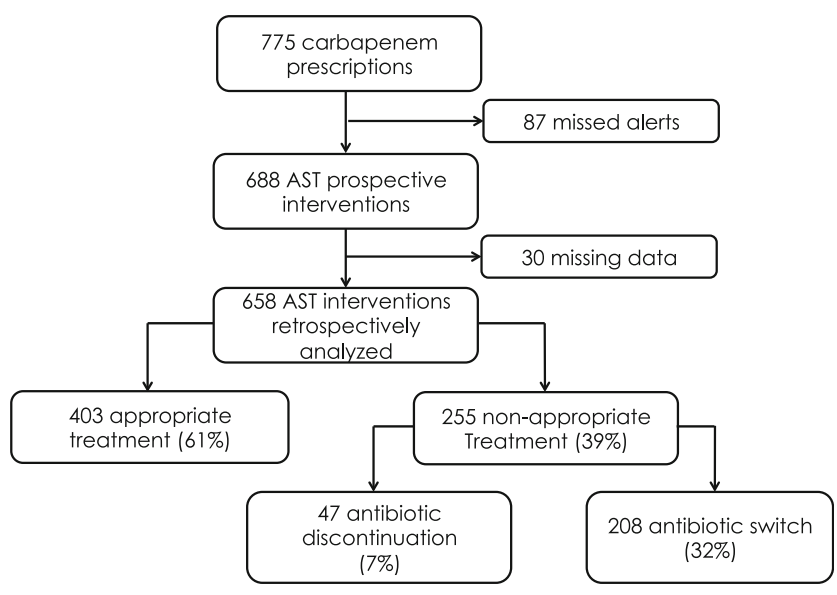

Fig. 2 Flow chart of carbapenem prescriptions included in the study. $A S T$, antibiotic stewardship team

especially for immunocompromised patients. Carbapenem regimens have been prescribed with a proper dosage and/or administration routine in more than $90 \%$ of cases. However, more than one-third of prescriptions were considered nonappropriate and have been discontinued following AST recommendation, mostly in paediatric wards.

\section{Interpretation}

Characteristics of our population on carbapenem therapy are consistent with previous studies, as they display comorbidities, carry ESBLE, and are often hospitalized in ICUs [19]. Besides, lung and urinary tract are commonly the major sites of infections, and almost all carbapenem regimens are prescribed for healthcare-associated infections [17, 19]. These observations are consistent with known risk factors for ESBLE carriage and infections, such as hospitalization in ICUs, devices such as central venous cathether, previous hospitalization, and antibiotic therapy [33-35]. However, we observed differences between adult and paediatric wards. First, the highest carbapenem use when standardized to 1000 patient days was observed in adult wards. It may be explained in part by the characteristics of our tertiary hospital whose adult wards (Departments of Infectious diseases, Nephrology and Transplantation, Haematology, and adult ICU) admit almost exclusively immunocompromised patients $(85 \%$, vs. $34 \%$ in paediatric wards). The higher rate of lower respiratory tract infections and causal pathogens as K. pneumoniae or Pseudomonas spp. observed in children is clearly related to the high number of patients hospitalized in paediatric ICUs, thus susceptible to ventilator-associated pneumonia. The more frequent use of ertapenem in adult wards is in accordance with current practice in adults, as it can be administered once a day [36]. We observed a sub-optimal dosage and administration routine more often in adult wards, which might be explained by a higher frequency of kidney failure in adults and subsequent difficulties to provide optimal dosage [37]. However, no measurement of serum carbapenem concentration was assessed in our study to confirm this hypothesis.

In our study, only one-third of carbapenem prescriptions were initiated after microbiological documentation; the major rate of empirical carbapenem initiation observed in both adult and paediatric wards by the prescribers reveals their reluctance of narrower spectrum antimicrobial agents for antibiotic initiation despite the absence of ESBLE colonization, most of the time because of the patients' underlying severe diseases, especially innate or acquired immune deficiency. This suggests poor adherence to predictive tools for ESBLE infections and subsequent recommendations for empirical carbapenem prescription $[38,39]$. Among the documented infections, $61 \%$ of pathogens were susceptible to other antibiotics according to antibiotic susceptibility testing. Beta-lactam/beta-lactamase inhibitors were showed to be an acceptable alternative in some studies, even in febrile aplasia/neutropenia [40-43]. A recent study suggests that, regarding definitive therapy for infections caused by 3rd generation cephalosporin-resistant Enterobacteriaceae, overall mortality might be lower for patients receiving carbapenems compared with those receiving piperacillin-tazobactam [5]. These discrepancies prompt us to consider the studied population, the primary outcome, and the type of infections in such studies, as treatment success also depends on $\mathrm{PK} / \mathrm{PD}$ properties, the site of infection, and antibiotic diffusion [44-46]. Carbapenems might be preferable for deep-seated documented infections with ESBLE, and alternatives might be easier to find in community-onset ESBLE infections and urinary tract infections [3]. All these data suggest the need for a consensual strategy to help clinicians for empirical therapy, especially during healthcare-associated infections in patients with severe underlying diseases.

In our tertiary hospital, the AST aims at improving infectious disease management and contain non-appropriate carbapenem prescriptions. This study revealed that more than onethird of carbapenems prescriptions were considered nonappropriate by the AST and its intervention led to either discontinuation or de-escalation of carbapenem. Most of these non-appropriate prescriptions were documented (76\%). These data confirm the potential impact of carbapenem stewardship, through a strategy combining diagnostic and therapeutic advices during systematic visits of AST in all wards and post-prescription review with rapid feedback [17, 18]. Development of AST in hospitals shows a better control antibiotic prescriptions and help physicians to improve health care and consider alternative antibiotics [14, 15] [HAS, 2019 \#57]. It implies, together with clinical experience, getting all data needed to assess and reduce the antibiotic spectrum, complying to guidelines, and considering the microbial ecology of the patient and wards, predictive PK-PD, benefit-to-risk ratio for the patient, and the cost. Recent implementation of antibiotic 
Table 3 Univariate analysis of factors associated with non-appropriate carbapenem prescriptions following AST review $(N=658)$

\begin{tabular}{|c|c|c|c|c|c|}
\hline Factors $(\%)$ & $N$ & Appropriate & Non-appropriate & Odds ratio $(95 \% \mathrm{CI})$ & $p$ \\
\hline \multicolumn{6}{|l|}{ Prescription characteristics } \\
\hline \multicolumn{6}{|l|}{ Paediatric prescriptions } \\
\hline Yes & 348 & $197(57)$ & $151(43)$ & $1.5(1.1-2.1)$ & 0.01 \\
\hline No & 310 & $206(67)$ & $104(33)$ & Reference & \\
\hline \multicolumn{6}{|l|}{ Empirical prescription } \\
\hline Yes & 452 & $273(60)$ & $179(40)$ & $1.0(0.7-1.5)$ & 0.80 \\
\hline No & 174 & $107(61)$ & $67(39)$ & Reference & \\
\hline Wards prescribing carbapenems & & & & & 0.07 \\
\hline Departments of medicine & 259 & $165(64)$ & $94(36)$ & $0.9(0.39-1.2)$ & \\
\hline Departments of surgery & 29 & $15(52)$ & $14(48)$ & $1.4(0.7-3)$ & \\
\hline Department of haematology/immunology & 88 & $61(69)$ & $27(31)$ & $0.7(0.4-1.1)$ & \\
\hline Neonatal intensive care unit & 47 & $22(47)$ & $25(53)$ & $1.7(1.1-3.2)$ & \\
\hline Intensive care units & 235 & $140(60)$ & $95(40)$ & Reference & \\
\hline \multicolumn{6}{|l|}{ Patient characteristics } \\
\hline \multicolumn{6}{|l|}{ Comorbidities } \\
\hline Yes & 615 & $375(61)$ & $194(39)$ & $1.4(0.6-3.2)$ & 0.39 \\
\hline No & 29 & $20(69)$ & $9(31)$ & Reference & \\
\hline \multicolumn{6}{|l|}{ Immune deficiency } \\
\hline Yes & 370 & $243(66)$ & $127(34)$ & $0.7(0.5-0.9)$ & 0.009 \\
\hline No & 274 & $152(56)$ & $122(44)$ & Reference & \\
\hline \multicolumn{6}{|l|}{ Known carriage of ESBLE } \\
\hline Yes & 331 & $226(68)$ & $105(32)$ & $0.5(0.4-0.7)$ & $<0.001$ \\
\hline No & 308 & $162(53)$ & $146(47)$ & Reference & \\
\hline \multicolumn{6}{|l|}{ Infection characteristics } \\
\hline \multicolumn{6}{|l|}{ Documented infection } \\
\hline Yes & 464 & $270(58)$ & $194(42)$ & $1.6(1.1-2.2)$ & 0.01 \\
\hline No & 194 & $133(69)$ & $61(31)$ & Reference & \\
\hline \multicolumn{6}{|l|}{ Septic shock } \\
\hline Yes & 63 & $46(73)$ & $17(27)$ & $0.6(0.3-0.9)$ & 0.04 \\
\hline No & 595 & $357(60)$ & $238(40)$ & Reference & \\
\hline \multicolumn{6}{|l|}{ Healthcare-associated infection } \\
\hline Yes & 548 & $337(62)$ & 211 (39) & $1.08(0.7-1.7)$ & 0.74 \\
\hline No & 98 & $62(63)$ & $37(36)$ & Reference & \\
\hline
\end{tabular}

stewardship programs is described to be associated with a significant decrease of carbapenem prescriptions [15, 21-23, 47, 48].

Our data also reveal that despite a lower DOT/1000 patient days, hospitalization in a paediatric ward, and NICU in particular, is associated with non-appropriateness. However guidelines for carbapenem use are common for both types of wards in our hospital. Children, neonates in particular, are known to be more exposed to antibiotics with a less proportion with confirmed infection $[49,50]$. This might be explained by a more challenging interpretation of clinical symptoms, risk factors, and biomarkers in children. Children are also often considered "vulnerable" by clinicians, and decision of a deescalation may be difficult to make [23]. Our study highlights that, despite common stewardship targets, tailored stewardship interventions considering the patients' specificities and issues, as well as clinicians' practice, perceptions, and attitudes, are a pre-requisite for their success [51].

\section{Limitations}

The major limit of this study is the lack of exhaustiveness for data collection by the AST compared to pharmacy alerts, which might be due to a lack of time dedicated to carbapenem surveillance, and the subsequent need of a retrospective review of the database. The surveillance and data collection was prospective, but all data were retrospectively reviewed for the study, leading to missing data. For example, we could 
not assess how often carbapenems were part of a en "escalation" strategy. We also observed that the median time to intervention of AST was longer than expected ( $>48 \mathrm{~h}$ ); these findings highlight that dedicating the necessary human resources to AST is also paramount for a fully successful prescription-control program. Finally, this study could not clearly measure the impact of a carbapenem stewardship program, as there was no comparator; we could not exclude that clinicians would have modified carbapenem therapy even without the intervention of the AST. Besides, we did not evaluate any correlation between AST intervention and changes in prevalence of antibiotic resistance; indeed, there are still concerns that reduction in consumption of some classes of antibiotics can result in an increase in other classes with possible similar deleterious ecological effects, and the impact of a reduction of treatment duration is still unclear [30,52].

\section{Conclusion}

Carbapenem therapy was generally initiated in severely ill patients or with risk factors of drug resistance. However, more than one-third of carbapenem prescriptions were considered non-appropriate by the AST, mostly in paediatric wards, with an indication for discontinuation or de-escalation. This work resulted in the implementation of local guidelines for carbapenem prescriptions. The study suggests that AST intervention is essential to better control paediatric antibiotic use and subsequent antimicrobial resistance.

Authors' contribution JT, MP, JRZ, and OL conceived the study. JP, PP, JFC, and JT designed the study. JP, PP, BP, JFC, and JT collected and were responsible for the data. PF and JRZ participated to data analysis. P, $\mathrm{PP}, \mathrm{BP}, \mathrm{OL}, \mathrm{JFC}$, and JT participated in patient care, investigation, and data collection. JT and JFC performed the statistical analysis. EB performed the microbiology analyses. JP and JT wrote the first draft of the manuscript. All authors drafted the manuscript for important intellectual content, contributed to the revision of the final version of the manuscript, and approved the final version submitted.

Funding This study was carried out as part of our routine work.

Data availability Data will be made available on reasonable request.

\section{Compliance with ethical standards}

Competing interests P.F. has received research grants (to his institution) from the French National Agency for AIDS Research (ANRS) and honoraria and travel grants from ViiV Healthcare, Bristol-Myers Squibb, Janssen Cilag, Gilead Sciences, Pfizer, Medtronic, and MSD France for participation in advisory boards, educational programs, and conferences, outside the submitted work. All other authors report no potential conflicts.

Ethical approval, consent to participate, and consent for publication Participants provided informed consent for the anonymous use of their clinical and biological data for biomedical research (For paediatric patients, informed consent was provided by parents/guardians). This study was reviewed and approved by the Necker Hospital Institutional Review Board (Registration number in the registry of the Assistance Publique - Hôpitaux de Paris: 2020 0117181406). All data processing and storage comply with the General Data Protection Regulation (GDPR) and ethical standards of the National Research Committee.

\section{References}

1. Hawkey PM (2015) Multidrug-resistant Gram-negative bacteria: a product of globalization. J Hosp Infect 89(4):241-247

2. Santé-Publique-France. Infections associées aux soins, résistance aux antibiotiques et consommation d'antibiotiques : données des réseaux de surveillance Raisin 2018 [Available from: https:// www.santepubliquefrance.fr/maladies-et-traumatismes/infectionsassociees-aux-soins-et-resistance-aux-antibiotiques/infectionsassociees-aux-soins/documents/rapport-synthese/infectionsassociees-aux-soins-resistance-aux-antibiotiques-etconsommation-d-antibiotiques-donnees-des-reseaux-desurveillance-raisin. Accessed 1 Jun 2020

3. Toubiana J, Timsit S, Ferroni A, Grasseau M, Nassif X, Lortholary $\mathrm{O}$ et al (2016) Community-onset extended-spectrum betalactamase-producing Enterobacteriaceae invasive infections in children in a university hospital in France. Medicine. 95(12):e3163

4. Delory T, De Pontfarcy A, Emirian A, About F, Berdougo B, BrunBuisson $C$ et al (2013) Impact of a program combining preauthorization requirement and post-prescription review of carbapenems: an interrupted time-series analysis. Eur J Clin Microbiol Infect Dis 32(12):1599-1604

5. Harris PNA, Tambyah PA, Lye DC, Mo Y, Lee TH, Yilmaz M et al (2018) Effect of piperacillin-tazobactam vs meropenem on 30-day mortality for patients with E coli or Klebsiella pneumoniae bloodstream infection and ceftriaxone resistance: a randomized clinical trial. Jama. 320(10):984-994

6. Turner PJ (2005) Extended-spectrum beta-lactamases. Clin Infect Dis 41(Suppl 4):S273-S275

7. Birgy A, Cohen R, Levy C, Bidet P, Courroux C, Benani M et al (2012) Community faecal carriage of extended-spectrum betalactamase-producing Enterobacteriaceae in French children. BMC Infect Dis 12:315

8. Goulenok T, Ferroni A, Bille E, Lecuyer H, Join-Lambert O, Descamps $P$ et al (2013) Risk factors for developing ESBL E. coli: can clinicians predict infection in patients with prior colonization? J Hosp Infect 84(4):294-299

9. Nicolas-Chanoine MH, Gruson C, Bialek-Davenet S, Bertrand X, Thomas-Jean F, Bert F et al (2013) 10-Fold increase (2006-11) in the rate of healthy subjects with extended-spectrum beta-lactamaseproducing Escherichia coli faecal carriage in a Parisian check-up centre. J Antimicrob Chemother 68(3):562-568

10. Reuland EA, Overdevest IT, Al Naiemi N, Kalpoe JS, Rijnsburger MC, Raadsen SA et al (2013) High prevalence of ESBL-producing Enterobacteriaceae carriage in Dutch community patients with gastrointestinal complaints. Clin Microbiol Infect 19(6):542-549

11. Barbier F, Bailly S, Schwebel C, Papazian L, Azoulay E, Kallel H et al (2018) Correction to: Infection-related ventilator-associated complications in ICU patients colonised with extended-spectrum beta-lactamase-producing Enterobacteriaceae. Intensive Care Med 44(7):1200-1202

12. Armand-Lefevre L, Angebault C, Barbier F, Hamelet E, Defrance G, Ruppe E et al (2013) Emergence of imipenem-resistant gramnegative bacilli in intestinal flora of intensive care patients. Antimicrob Agents Chemother 57(3):1488-1495 
13. santé A-Andsdmedpd. Evolution des consommations d'antibiotiques en France entre 2000 et 2015 - Point d'Information - ANSM 2018 [Available from: http://ansm.sante. fr/S-informer/Points-d-information-Points-d-information/ Evolution-des-consommations-d-antibiotiques-en-France-entre2000-et-2015-Point-d-Information. Accessed 1 Jun 2020

14. Baur D, Gladstone BP, Burkert F, Carrara E, Foschi F, Dobele S et al (2017) Effect of antibiotic stewardship on the incidence of infection and colonisation with antibiotic-resistant bacteria and Clostridium difficile infection: a systematic review and meta-analysis. Lancet Infect Dis 17(9):990-1001

15. Davey P, Marwick CA, Scott CL, Charani E, McNeil K, Brown E et al (2017) Interventions to improve antibiotic prescribing practices for hospital inpatients. Cochrane Database Syst Rev 2:CD003543

16. Howard P, Pulcini C, Levy Hara G, West RM, Gould IM, Harbarth $S$ et al (2015) An international cross-sectional survey of antimicrobial stewardship programmes in hospitals. J Antimicrob Chemother 70(4):1245-1255

17. Canoui E, Gauzit R, Bruneau A, Alviset S, Hays C, Charpentier J et al (2018) Assessment of appropriate carbapenem prescribing in stewardship programmes. J Hosp Infect 100(3):277-279

18. Fujibayashi A, Niwa T, Takeichi S, Suzuki K, Ohta H, Niwa A et al (2019) Clinical impact of a prospective audit with intervention and feedback without carbapenem restriction in patients receiving carbapenem injection. Int J Clin Pract 73(1):e13262

19. Lefebure A, Papy E, Rioux C, Diamantis S, Armand-Lefevre L, Longuet $\mathrm{P}$ et al (2015) Audit of carbapenem prescriptions comparing 2 assessment periods. Med Mal Infect 45(7):273-278

20. Robson SE, Cockburn A, Sneddon J, Mohana A, Bennie M, Mullen $\mathrm{AB}$ et al (2018) Optimizing carbapenem use through a national quality improvement programme. J Antimicrob Chemother 73(8): 2223-2230

21. Hemkens LG, Saccilotto R, Reyes SL, Glinz D, Zumbrunn T, Grolimund $\mathrm{O}$ et al (2017) Personalized prescription feedback using routinely collected data to reduce antibiotic use in primary care: a randomized clinical trial. JAMA Intern Med 177(2):176-183

22. Horikoshi Y, Suwa J, Higuchi H, Kaneko T, Furuichi M, Aizawa Y et al (2017) Sustained pediatric antimicrobial stewardship program with consultation to infectious diseases reduced carbapenem resistance and infection-related mortality. Int J Infect Dis 64:69-73

23. Patel SV, Vergnano S (2018) The impact of paediatric antimicrobial stewardship programmes on patient outcomes. Curr Opin Infect Dis 31(3):216-223

24. Seah XF, Ong YL, Tan SW, Krishnaswamy G, Chong CY, Tan NW et al (2014) Impact of an antimicrobial stewardship program on the use of carbapenems in a tertiary women's and children's hospital, Singapore. Pharmacotherapy 34(11):1141-1150

25. Leclercq R, Canton R, Brown DF, Giske CG, Heisig P, MacGowan AP et al (2013) EUCAST expert rules in antimicrobial susceptibility testing. Clin Microbiol Infect 19(2):141-160

26. Morris AM (2014) Antimicrobial stewardship programs: appropriate measures and metrics to study their impact. Curr Treat Options Infect Dis 6(2):101-112

27. Horan TC, Andrus M, Dudeck MA (2008) CDC/NHSN surveillance definition of health care-associated infection and criteria for specific types of infections in the acute care setting. Am J Infect Control 36(5):309-332

28. Dellinger RP, Levy MM, Rhodes A, Annane D, Gerlach H, Opal SM et al (2013) Surviving sepsis campaign: international guidelines for management of severe sepsis and septic shock: 2012. Crit Care Med 41(2):580-637

29. Harbarth S, Nobre V, Pittet D (2007) Does antibiotic selection impact patient outcome? Clin Infect Dis 44(1):87-93

30. Weiss E, Zahar JR, Garrouste-Orgeas M, Ruckly S, Essaied W, Schwebel C et al (2016) De-escalation of pivotal beta-lactam in ventilator-associated pneumonia does not impact outcome and marginally affects MDR acquisition. Intensive Care Med 42(12): 2098-2100

31. Barlam TF, Cosgrove SE, Abbo LM, MacDougall C, Schuetz AN, Septimus EJ et al (2016) Executive summary: implementing an antibiotic stewardship program: guidelines by the Infectious Diseases Society of America and the Society for Healthcare Epidemiology of America. Clin Infect Dis 62(10):1197-1202

32. Souverein D, Euser SM, van der Reijden WA, Herpers BL, Kluytmans J, Rossen JWA et al (2017) Clinical sensitivity and specificity of the Check-Points Check-Direct ESBL Screen for BD MAX, a real-time PCR for direct ESBL detection from rectal swabs. J Antimicrob Chemother 72(9):2512-2518

33. Freeman JT, McBride SJ, Nisbet MS, Gamble GD, Williamson DA, Taylor SL et al (2012) Bloodstream infection with extendedspectrum beta-lactamase-producing Enterobacteriaceae at a tertiary care hospital in New Zealand: risk factors and outcomes. Int J Infect Dis 16(5):e371-e374

34. Marchaim D, Gottesman T, Schwartz O, Korem M, Maor Y, Rahav G et al (2010) National multicenter study of predictors and outcomes of bacteremia upon hospital admission caused by Enterobacteriaceae producing extended-spectrum beta-lactamases. Antimicrob Agents Chemother 54(12):5099-5104

35. Rodriguez-Bano J, Lopez-Cerero L, Navarro MD, Diaz de Alba P, Pascual A et al (2008) J Antimicrob Chemother 62(5):1142-1149

36. Trad MA, Zhong LH, Llorin RM, Tan SY, Chan M, Archuleta S et al (2017) Ertapenem in outpatient parenteral antimicrobial therapy for complicated urinary tract infections. J Chemother 29(1):2529

37. Thalhammer F, Horl WH (2000) Pharmacokinetics of meropenem in patients with renal failure and patients receiving renal replacement therapy. Clin Pharmacokinet 39(4):271-279

38. Goodman KE, Lessler J, Cosgrove SE, Harris AD, Lautenbach E, Han JH et al (2016) A clinical decision tree to predict whether a bacteremic patient is infected with an extended-spectrum betalactamase-producing organism. Clin Infect Dis 63(7):896-903

39. Tumbarello M, Trecarichi EM, Bassetti M, De Rosa FG, Spanu T, Di Meco E et al (2011) Identifying patients harboring extendedspectrum-beta-lactamase-producing Enterobacteriaceae on hospital admission: derivation and validation of a scoring system. Antimicrob Agents Chemother 55(7):3485-3490

40. Gutierrez-Gutierrez B, Perez-Galera S, Salamanca E, de Cueto M, Calbo E, Almirante B et al (2016) A multinational, preregistered cohort study of beta-lactam/beta-lactamase inhibitor combinations for treatment of bloodstream infections due to extended-spectrumbeta-lactamase-producing Enterobacteriaceae. Antimicrob Agents Chemother 60(7):4159-4169

41. Khoo AL, Zhao YJ, Teng M, Ying D, Jin J, Chee YL et al (2018) Evaluation of a risk-guided strategy for empirical carbapenem use in febrile neutropenia. Int J Antimicrob Agents 52(3):350-357

42. Sheu CC, Lin SY, Chang YT, Lee CY, Chen YH, Hsueh PR (2018) Management of infections caused by extended-spectrum betalactamase-producing Enterobacteriaceae: current evidence and future prospects. Expert Rev Anti-Infect Ther 16(3):205-218

43. Walker KJ, Lee YR, Klar AR (2018) Clinical outcomes of extended-spectrum beta-lactamase-producing Enterobacteriaceae infections with susceptibilities among levofloxacin, cefepime, and carbapenems. Can J Infect Dis Med Microbiol 2018:3747521

44. Prevel R, Berdai D, Boyer A (2019) Antibiotics for ceftriaxoneresistant gram-negative bacterial bloodstream infections. Jama. 321(6):613

45. Rodriguez-Bano J, Gutierrez-Gutierrez B, Kahlmeter G (2019) Antibiotics for ceftriaxone-resistant gram-negative bacterial bloodstream infections. Jama. 321(6):612-613

46. Tamma PD, Rodriguez-Bano J (2017) The use of noncarbapenem beta-lactams for the treatment of extended-spectrum beta-lactamase infections. Clin Infect Dis 64(7):972-980 
47. Faraone A, Poggi A, Cappugi C, Tofani L, Riccobono E, Giani T et al (2020) Inappropriate use of carbapenems in an internal medicine ward: impact of a carbapenem-focused antimicrobial stewardship program. Eur J Intern Med 78:50-57

48. Mani NS, Lan KF, Jain R, Bryson-Cahn C, Lynch JB, Krantz EM et al (2020) Post-prescription review with threat of infectious disease consultation and sustained reduction in meropenem use over four years. Clin Infect Dis

49. Cantey JB, Baird SD (2017) Ending the culture of culture-negative sepsis in the neonatal ICU. Pediatrics 140(4):e20170044. https:// doi.org/10.1542/peds.2017-0044

50. Fjalstad JW, Stensvold HJ, Bergseng H, Simonsen GS, Salvesen B, Ronnestad AE et al (2016) Early-onset sepsis and antibiotic exposure in term infants: a nationwide population-based study in Norway. Pediatr Infect Dis J 35(1):1-6

51. Patel PK (2019) One size doesn't fit all-stewardship interventions need to be tailored in large healthcare systems. Clin Infect Dis

52. Woerther PL, Lepeule R, Burdet C, Decousser JW, Ruppe E, Barbier F (2018) Carbapenems and alternative beta-lactams for the treatment of infections due to extended-spectrum betalactamase-producing Enterobacteriaceae: what impact on intestinal colonisation resistance? Int J Antimicrob Agents 52(6):762-770

Publisher's note Springer Nature remains neutral with regard to jurisdictional claims in published maps and institutional affiliations. 